\title{
The spacing effect in the free recall of homogeneous lists: Present and accounted for
}

\author{
THOMAS C. TOPPINO, YOKO HARA, and JESSICA HACKMAN \\ Villanova University, Villanova, Pennsylvania
}

\begin{abstract}
Memory performance nearly always improves as a function of the spacing between repetitions. However, previous studies indicated that college students exhibited no spacing effect in the free recall of lists composed exclusively of words sampled from a single semantic category. We explored this puzzling phenomenon in two experiments. We found that the spacing effect in free recall can occur with homogeneous lists. Most interestingly, the effect seems to depend on the number of items (lag) separating spaced repetitions. Short lags between spaced repetitions yield a spacing effect, whereas longer lags do not.
\end{abstract}

The spacing effect refers to the finding that spaced repetitions separated by time and/or other events are remembered better than massed repetitions that occur in immediate succession. Although no explanation of the spacing effect has gained general acceptance (Dempster, 1996), most accounts fall into one of three categories depending on the type of mechanism that is assumed to produce the effect. ${ }^{1}$ According to deficient-processing theories (e.g., Challis, 1993; Jacoby, 1978; Shaughnessy, Zimmerman, \& Underwood, 1972), the spacing effect results from inadequate processing of massed repetitions relative to spaced repetitions. Encoding-variability theorists (e.g., Bower, 1972; Glenberg, 1979) hypothesize that spaced repetitions are more likely than massed repetitions to undergo variable encoding, which facilitates memory by increasing the number of effective retrieval routes. And, from the perspective of study-phase-retrieval theories (e.g., Braun \& Rubin, 1998; Thios \& D'Agostino, 1976), retrieval of the first presentation at the time of the second is essential, with the beneficial effect being greater for spaced than for massed repetitions.

Despite the lack of theoretical agreement, the spacing effect is a remarkably robust phenomenon that has been demonstrated in an unusually wide variety of circumstances (see Dempster, 1996, for a review). Given the relative ubiquity of the phenomenon, any set of conditions in which the spacing effect is reliably attenuated may offer an opportunity to gain fresh theoretical insights. In the present experiments, we examined a failure to obtain a spacing effect in free recall that originally was reported by Elmes, Dye, and Herdelin (1983) and that subsequently was replicated and extended by Kahana and Greene (1993).

We thank Amy Drummond, Yvonne Leslie, and Jessica Blundell for their assistance in collecting the data for Experiment 1. Correspondence should be addressed to T. C. Toppino, Department of Psychology, Villanova University, 800 Lancaster Ave., Villanova, PA 19085 (e-mail: thomas.toppino@villanova.edu).
Elmes et al. (1983) were interested in the influence of affect on the spacing effect. The important element of their findings for our purposes was that, when lists contained unrelated, affectively neutral words, Elmes et al. (1983) found that free recall was better for spaced repetitions than for massed repetitions, whereas when lists were affectively homogeneous (i.e., composed of all "good" words or all "bad" words), the spacing effect was eliminated.

Although other aspects of the findings reported by Elmes et al. (1983), in conjunction with subsequent work (Elmes, Chapman, \& Selig, 1984), suggested a complex relationship between affect and the spacing of repetitions, the elimination of the spacing effect with affectively homogeneous lists may have had more to do with the lists being homogeneous than with their affective characteristics. Kahana and Greene (1993) replicated the findings of Elmes et al. (1983), but demonstrated that the spacing effect in free recall also was eliminated with homogeneous lists that were affectively neutral. Thus, the spacing effect in free recall was eliminated when list words consisted entirely of four-footed animals or entirely of occupations. Kahana and Greene suggested that the findings of Elmes et al. (1983) with affectively loaded words was simply a particular instance of a more general phenomenon related to homogeneous lists.

Kahana and Greene (1993) noted that this phenomenon was "generally unaddressed" by theories of the spacing effect and offered a post hoc account based on Greene's (1989) two-process theory. According to this view, repetition of an item is assumed to trigger automatic studyphase retrieval of its first presentation and automatic encoding of new contextual elements that reflect contex tual change since the earlier presentation. When the spacing between repetitions is greater, there will be more contextual change from one presentation to the next, and more new contextual elements will be encoded. Because free recall typically depends heavily on contextual cues, the greater variety of contextual elements stored with spaced 
repetitions generally affords a retrieval advantage for these items over massed repetitions. In the case of free recall of homogeneous lists, however, Kahana and Greene hypothesized that retrieval may be guided by a salient category cue (i.e., the feature shared by all list items) rather than by contextual cues. They reasoned that this would neutralize the effects of contextual variability and eliminate the spacing effect in free recall.

\section{EXPERIMENT 1}

Our first experiment was designed to provide a simple, straightforward test of Kahana and Greene's (1993) interpretation of the phenomenon in question. We compared the free recall of massed versus spaced repetitions that were presented in a homogeneous list. However, whereas Elmes et al. (1983) and Kahana and Greene had presented each repeated item two times, we presented each repeated item three times. Kahana and Greene's hypothesis should not be sensitive to the frequency with which repeated items are presented. Therefore, their hypothesis leads to the prediction that we should replicate previous results by failing to obtain a spacing effect. However, we thought there was a chance that the use of thrice-presented items might yield different results. The magnitude of the spacing effect sometimes increases with the frequency of repetitions (e.g., Shaughnessy et al., 1972; Underwood, 1970), and, if nothing else, we thought a more potent spacing effect might be easier to detect.

\section{Method}

Participants, Procedure, and Design. Participants were 54 introductory psychology students from Villanova University. Each participant viewed a single list of words presented one item at a time in the center of a computer screen. Each item appeared for $2.5 \mathrm{sec}$ with $.5 \mathrm{sec}$ of blank screen separating successive presentations. Within the list, items varied with respect to their number of presentations and the spacing between repetitions. There were three critical subsets of items: once-presented items, massed-repetition items, and spaced-repetition items. Prior to list presentation, participants were instructed to study the sequence of words for a subsequent memory test. Immediately after list presentation, participants were administered a free-recall test in which they wrote down as many words from the list as they could remember.

Materials. Two list structures that determined the assignment of within-list conditions to serial positions were independently generated with the requirement that they both conform to the following restrictions. Both structures contained slots or places for 61 words. In addition, the first eight and last eight positions were reserved for once-presented items that served as primacy and recency buffers, respectively. The middle portion of the list structures contained slots for 10 once-presented filler words, 5 once-presented experimental words, and 10 repeated words that were each presented three times. Five of the repeated words represented massed repetitions, for which all three presentations occurred in immediate succession. The other five repeated words represented spaced repetitions, for which successive occurrences were separated by a lag of three or four other item presentations. In an effort to avoid confounding by extended recency effects that sometimes affect free-recall performance (e.g., Underwood, 1969), both list structures were constructed so that the mean serial position of the final occurrences of items in the massed-repetition and spaced-repetition conditions were equated with one another and with the mean serial position of the once-presented experimental items.

Six lists (three using each list structure) were generated from a set of 41 words selected from the "four-footed animal" category of Battig and Montague's (1969) norms. To create the first list with each structure, words were randomly assigned to slots reserved for primacy buffers, recency buffers, fillers, once-presented experimental items, massed repetitions, and spaced repetitions, respectively. To generate the remaining two lists with each structure, words originally assigned to serve as once-presented experimental items, massed repetitions, and spaced repetitions were rotated among the three conditions using a Latin-square principle. Thus, across the three lists constructed from each structure, the same set of items served equally often as once-presented experimental items, massed-repetition items, and spaced-repetition items. An equal number of participants was administered each of the six lists.

\section{Results}

Because frequency of occurrence (one vs. three presentations) and the spacing between repetitions (massed vs. spaced) are conceptually distinct variables, their effects were assessed in separate analyses. Significance was set at $p=.05$ for all tests.

In the first analysis, a $t$ test for related means was conducted on the mean percentage of correct recall for the once-presented experimental items and for thrice-presented items (combined massed and spaced repetitions). Results revealed that recall was significantly better for repeated items $(M=62.04 \%, S E=2.05)$ than for once-presented items $(M=36.30 \%, S E=3.35)[t(53)=9.18]$. In the second analysis, another $t$ test for related means revealed a significant spacing effect such that spaced repetitions $(M=68.89 \%, S E=3.16)$ were recalled significantly better than massed repetitions $(M=55.19 \%, S E=2.94)[t(53)=$ 3.04].

\section{Discussion}

Kahana and Greene (1993) explained why they failed to obtain a spacing effect in free recall with homogeneous lists as follows: (1) The spacing effect in free recall is a result of contextual variability, and (2) participants recalling a homogeneous list use a salient category cue to guide retrieval and, therefore, are unaffected by contex tual variability. Our results are not consistent with Kahana and Greene's hypothesis. We obtained a clear spacing effect in free recall following the presentation of a homogeneous list. Of course, we presented repeated items three times, whereas in previous experiments using homogeneous lists, repeated items were presented only twice. However, Kahana and Greene's hypothesis does not explain why the frequency of repetitions would influence whether or not a spacing effect is obtained.

\section{EXPERIMENT 2}

In hopes of explaining the discrepancy between the results of Experiment 1 and the findings previously reported by Elmes et al. (1983) and Kahana and Greene (1993), we examined the salient methodological difference among the studies. The most obvious difference, of course, is that 
we repeated items three times each, whereas in the previous work, repeated items were presented twice. This alone could be the critical difference, but it is unclear why. In the introduction, we noted that the spacing effect is sometimes (though not always) greater with thricepresented items. To the extent that the spacing effect is greater, it might be more likely to be discerned amidst the noise of random variation. However, this hypothesis is unsatisfying for two reasons. First, it fails to explain why homogeneous lists minimize an effect that is normally very strong and robust with twice-presented items. Second, whereas this hypothesis might predict that by using thrice-presented items, one would be able to discern a spacing effect that was undetectable with twicepresented items, the effect would hardly be expected to become suddenly robust. However, rather than a relatively weak and tenuous effect, we obtained a very strong spacing effect in Experiment 1.

A second difference between our research and previous experiments is that we presented to-be-remembered items visually, whereas both Elmes et al. (1983) and Kahana and Greene (1993) used auditory presentation. However, this difference seems unlikely to account for the discrepant findings. Although there is at least one case in which the use of auditory presentation was associated with an otherwise unexplained failure to obtain the spacing effect (Waugh, 1963), presentation modality generally has had little influence on the phenomenon (e.g., Melton, 1970).

The third major difference involved the lag separating spaced repetitions. Elmes et al. (1983) and Kahana and Greene (1993) compared massed repetitions with spaced repetitions in which successive occurrences of an item were separated by a lag of seven or eight other presentations. In contrast, in our Experiment 1, successive occurrences of spaced-repetition items were separated by a lag of only three or four other presentations. Interestingly, for this difference to account for the discrepant findings, the shorter lags we used would have to have produced a larger spacing effect than the longer lags used by previous investigators. This would constitute a reversal of the usual finding, in which longer lags yield better performance than shorter lags (e.g., Melton, 1970) and would have potentially interesting theoretical implications. As we will discuss later, such a finding would be more easily reconciled with a study-phase retrieval account of the spacing effect than with deficient-processing or encoding-variability theories.

In the present experiment, we empirically examined the influence of all three of the methodological differences described above between our first experiment and the research that inspired it by Elmes et al. (1983) and Kahana and Greene (1993). We examined the effect of spacing repetitions on the free recall of homogeneous lists following study in three different conditions that differed in how repetitions were presented. In one case, repeated items were presented three times each with relatively short lags separating spaced repetitions. In the remaining two study conditions, repeated items were each presented twice. The latter two conditions differed with respect to whether the separation between spaced-repetition items involved relatively long, or relatively short, lags. Finally, we systematically varied the modality in which lists were presented.

We expected to replicate the results of Experiment 1 when study lists involved the visual presentation of thrice-presented items. We also expected to replicate the failure to obtain the spacing effect previously reported by Elmes et al. (1983) and by Kahana and Greene (1993) when study lists involved the auditory presentation of twice-presented items for which spaced repetitions were separated by relatively long lags. The remaining conditions were intended to clarify the source of these discrepant results. If the spacing effect in the free recall of homogeneous lists depends critically on the frequency of repetitions, a spacing effect would be expected in the thrice-presented conditions but not in any condition involving twice-presented items. If the critical variable, however, is the degree of spacing between occurrences of spaced-repetition items, a spacing effect would be expected both when repetitions occur three times each and when twice-presented spaced-repetition items are separated by relatively short lags. Finally, if the critical variable is presentation modality, a spacing effect would be expected in conditions for which list presentation is visual but not for those in which it is auditory.

\section{Method}

Participants and Design. Participants were 144 introductory psychology students at Villanova University. Twenty-four participants were assigned randomly in blocks of 6 to the six between-subjects conditions of a $3 \times 2 \times 3$ (frequency/lag condition $\times$ modality $\times$ repetition/spacing condition) mixed factorial design with the last factor varied within subjects. The three frequency/lag conditions were the thrice-presented condition, the twice-presented/long-lag condition, and the twice-presented/short-lag condition. Modality of list presentation was either visual or auditory. The within-list repetition/ spacing variable had three levels: once-presented items, massedrepetition items, and spaced-repetition items.

Materials. A total of six list structures were created independently, two for each frequency/lag condition. For the thrice-presented condition, structures contained slots for 56 presentations, whereas structures for the two twice-presented conditions contained slots for 46 presentations. In every structure, the first eight and the last eight positions were reserved for once-presented items, which served as primacy and recency buffers, respectively. The middle portion of all list structures contained slots for five experimental once-presented items, five massed-repetition items, five spacedrepetition items, and five once-presented filler items. Structures were generated so that successive occurrences of spaced-repetition items would be separated by three or four other presentations in the thrice-present ed condition, by eight other presentations in the twice-presented /long-lag condition, and by three other presentations in the twice-presented/ short-lag condition. ${ }^{2}$ As in Experiment 1, all list structures were created so that the sets of items representing the once-presented experimental condition, the massed-repetition condition, and the spaced-repetition condition, respectively, were equated with one another in terms of their mean serial position of final occurrence (or only occurrence, in the case of once-presented items). 
To-be-remembered items were selected from Battig and Montague's (1969) category norms. They consisted of 36 words selected from the "four-footed-animals" category and another 36 words selected from the "occupations" category. These words were used to create 18 different lists, 3 lists with each of the two list structures that had been created for each of the three frequency/lag conditions of the experiment. For each of the three frequency/lag conditions, the three lists generated from one list structure used words from the four-footed-animal category and the three lists generated from the other list structure used items from the occupations category. After the first list was created with each list structure, the remaining two lists were generated by using a Latin-square principle to rotate words among the three repetition/spacing conditions. In this way, the same set of items served equally often in each repetition/spacing condition. An equal number of participants received each of the six lists created for each of the three frequency/lag conditions.

Procedure. Procedure in the visual-presentation conditions was identical to that of Experiment 1. In the auditory-presentation conditions, the only difference was that lists, which had been recorded in a female voice, were presented via auditory tape.

\section{Results and Discussion}

Preliminary analyses indicated that, as expected, no reliable effects were attributable to the category from which to-be-remembered items were sampled (fourfooted animals vs. occupations). Therefore, the data were collapsed across category for all reported analyses.

In the first major analysis, we compared the mean percentage of correct free recall of once-presented and repeated items (massed and spaced repetitions combined). A $3 \times 2 \times 2$ (frequency/lag condition $\times$ modality $\times$ repetition condition) analysis of variance (ANOVA) with repeated measures on the last factor revealed only two sig- nificant effects. First, there was a significant effect of frequency/lag condition $\left[F(2,138)=3.16, M S_{\mathrm{e}}=394.14\right]$, reflecting the fact that the thrice-presented condition ( $M=46.25 \%)$ outperformed the twice-presented/long-lag condition $(M=40.73 \%)$ and the twice-presented/short-lag condition $(M=39.48 \%)$. Although the frequency/lagcondition $\times$ repetition interaction was not reliable, separate analyses for once-presented and repeated items, respectively, indicated that items presented three times in the thrice-presented condition enjoyed a performance advantage over items presented two times in the twicepresented conditions $\left[F(2,141)=3.58, M S_{\mathrm{e}}=268.17\right]$. However, the three frequency/lag conditions did not differ significantly on the recall of once-presented items $\left[F(2,141)=1.26, M S_{\mathrm{e}}=372.22\right]$. The second, and more important, finding from the overall ANOVA was that recall was better for repeated items $(M=53.19 \%)$ than for once-presented items $(M=31.11 \%)$. The main effect of repetition was significant $\left[F(1,138)=139.61, M S_{\mathrm{e}}=\right.$ $251.51]$ and was unqualified by an interaction with any other variable. Thus, if we fail to obtain a spacing effect in any condition, the finding cannot be attributed to a more general failure of repetition to influence performance.

The analysis of primary interest involved the effect of spacing repetitions. The percentage of correct free recall is presented in Figure 1, and these data were submitted to a $3 \times 2 \times 2$ (frequency/lag condition $\times$ modality $\times$ spacing) ANOVA with repeated measures on the final factor. There was some tendency for recall to be better following visual presentation $(M=55.56 \%)$ than following auditory presentation $(M=50.84 \%)$, but the main

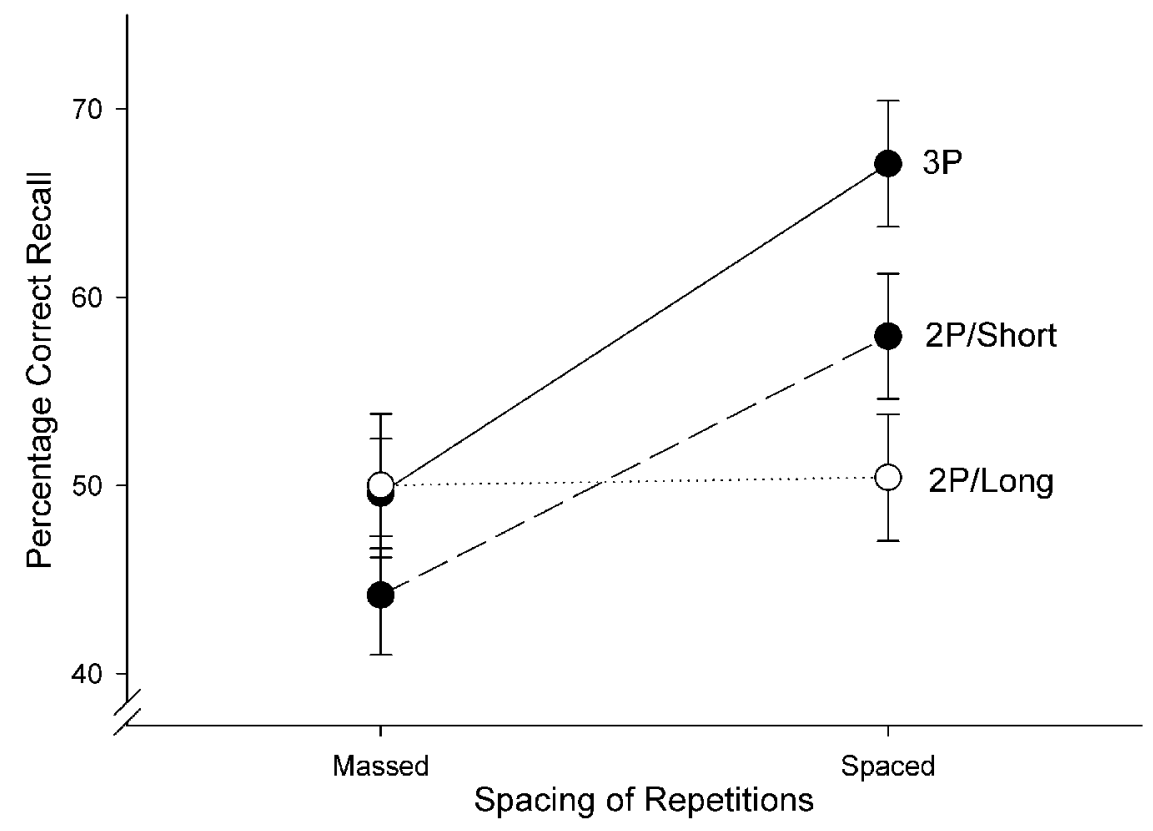

Figure 1. Mean percentage of correct free recall (with standard error bars) in Experiment 2 as a function of the spacing between repetitions and the three study conditions: thrice-presented (3P), twice-presented/short-lag (2P/Short), and twice-presented/long-lag (2P/Long). 
effect of modality fell short of significance $[F(1,138)=$ $\left.3.01, M S_{\mathrm{e}}=533.82\right]$. More importantly, modality did not interact with any other variable (all $F$ s $<1.44$ ). There was a main effect of frequency/lag condition $[F(2,138)=$ $\left.3.59, M S_{\mathrm{e}}=533.82\right]$, a main effect of spacing repetitions $\left[F(1,138)=15.17, M S_{\mathrm{e}}=528.26\right]$, and a significant spacing $\times$ frequency/lag-condition interaction $[F(2,138)=$ 3.66, $\left.M S_{\mathrm{e}}=528.26\right]$. To probe the interaction, the simple main effect of spacing was assessed for each frequency/lag condition. When repeated items were presented three times each, spaced repetitions were recalled better than massed repetitions $\left[F(1,138)=13.91, M S_{\mathrm{e}}=528.26\right]$, replicating the results of Experiment 1. For the twicepresented/long-lag condition, there was no hint of a spacing effect $\left[F(1,138)<1.00, M S_{\mathrm{e}}=528.26\right]$. Thus, when our study conditions closely corresponded to those of Elmes et al. (1983) and Kahana and Greene (1993), we replicated their failure to obtain a spacing effect in the free recall of homogeneous lists. The most telling finding, however, was a significant spacing effect in the twice-presented/short-lag condition $[F(1,138)=8.59$, $\left.M S_{\mathrm{e}}=528.26\right]$.

The crucial comparison in this experiment involves our two twice-presented conditions. It shows that short distributed lags improve performance relative to massed repetitions, but that longer lags do not. These findings indicate that previous studies (Elmes et al., 1983; Kahana \& Greene, 1993) failed to obtain a spacing effect in free recall of homogeneous lists because they inadvertently used a lag between occurrences of spaced repetitions that was too long. It seems likely that the use of short interpresentation lags between occurrences of spaced repetitions also accounts for the spacing effect we obtained with thrice-presented items, although the design of our experiment does not allow us to rule out the possibility of an independent contribution due to presentation frequency.

\section{GENERAL DISCUSSION}

Previous research (e.g., Elmes et al., 1983; Kahana \& Greene, 1993) indicated that the spacing effect was not obtained in free recall when list items were semantically homogeneous. Kahana and Greene suggested that the critical factor was the use of homogeneous lists per se. They speculated that, with these lists, retrieval may be based on the salient category cue shared by all list members, thereby bypassing contextually based retrieval, which was thought to mediate the spacing effect in most free-recall situations. However, this hypothesis does not seem tenable. The results of both of our experiments clearly demonstrate that a spacing effect can occur in the free recall of homogeneous lists.

Our research also clarified the conditions under which the spacing effect is and is not obtained. The results of Experiment 2 indicate that a critical factor is the length of the lag separating occurrences of spaced-repetition items. All of the earlier studies involving homogeneous lists had fortuitously employed spaced repetitions with a lag of seven or eight presentations separating occurrences. When we employed lags of a similar length, we replicated their failure to obtain a spacing effect. However, when we employed shorter lags between occurrences of spaced-repetition items, a strong spacing effect was demonstrated, both for twice- and thrice-presented words.

The fact that a relatively short lag between repetitions improved recall over massed repetitions, whereas a relatively long lag did not, represents a reversal of the usual finding in which longer lags are more effective than shorter ones (e.g., Melton, 1970). In terms of theory, this reversal seems more easily reconciled with a study-phase retrieval explanation of the spacing effect than with either deficient-processing or encoding-variability accounts, which have dominated the spacing effect literature.

The essence of both deficient-processing theories (e.g., Shaughnessy et al., 1972) and encoding-variability theories (e.g., Bower, 1972; Madigan, 1969) is that increased spacing is associated with changes in processing that should produce a monotonic increase in performance. Repetitions may become more fully or more adequately processed with increased spacing (deficientprocessing theories), or they become more likely to be differentially encoded, thereby increasing the probability of retrieval (encoding-variability theories). But, in either case, performance is predicted to improve until spacing has a maximal effect, after which the performance level should be maintained. In order to account for our results, these theories must incorporate a mechanism that allows shorter lags to produce better performance than longer lags and must relate its operation to the use of homogeneous lists. ${ }^{3}$

A study-phase retrieval account of the spacing effect seems to be more readily reconciled with our findings. According to this view, successful retrieval of an item's first presentation at the time of its later occurrence is necessary for the spacing effect to be obtained (e.g., Braun \& Rubin, 1998; Thios \& D'Agostino, 1976). However, as the spacing between repetitions increases, performance is influenced by two opposing processes: (1) The benefits of repetition become greater for items that undergo successful study-phase retrieval, and (2) the probability of successful study-phase retrieval decreases (Toppino \& Bloom, 2002). Performance initially improves with increases in spacing because study-phase retrieval is successful for most items, and the first process dominates. However, there must be a level of spacing at which performance peaks and then begins to decline, because the second process increasingly outweighs the first. The level of spacing at which this reversal occurs should depend on factors that influence the probability of successful study-phase retrieval.

In the case of homogeneous lists, high intralist similarity among items may interfere with successful studyphase retrieval by causing extensive proactive and retroactive interference (e.g., Wickens, 1972). Furthermore, 
the deleterious effect of interference on study-phase retrieval would be expected to become greater with increased spacing because the effects of interference are more pronounced with longer retention intervals (e.g., Wixted \& Rohrer, 1993). Thus, from the perspective of a study-phase retrieval theory, the use of homogeneous lists may establish the conditions under which relatively short lags produce a stronger spacing effect than longer lags, whereas the opposite might be expected in comparable lists involving unrelated words (e.g., Elmes et al., 1983).

Our results have clarified the conditions surrounding the curious absence of the spacing effect in the free recall of homogeneous lists that was reported in previous studies, but a related issue remains unresolved. Although Kahana and Greene (1993) obtained no spacing effect with homogeneous lists when memory was tested by free recall, they found a pronounced spacing effect under similar conditions when memory was tested by cuedmemory tasks (i.e., recognition in Experiments 4 and 5 and frequency discrimination in Experiment 6). When these findings of Kahana and Greene are considered in conjunction with the present results, they indicate that distributed lags that are long enough to eliminate the spacing effect in free recall of homogeneous lists may not be sufficient to eliminate the effect when cued-memory tasks are used. Why this is so remains unclear. It seems likely that somewhat different processes may contribute to the spacing effect in free recall and in cued-memory tasks, respectively, as suggested by Kahana and Greene (see also Greene, 1989). It also seems likely that further research with homogeneous lists may help clarify the nature of these different processes.

\section{REFERENCES}

Battig, W. F., \& Montague, W. E. (1969). Category norms of verbal items in 56 categories: A replication and extension of the Connecticut category norms. Journal of Experimental Psychology, 80 (3, Pt. 2), $1-46$.

Bower, G. H. (1972). Stimulus-sampling theory of encoding variability. In A. W. Melton \& E. Martin (Eds.), Coding processes in human memory (pp. 85-123). Washington, DC: Winston.

Braun, K., \& Rubin, D. C. (1998). The spacing effect depends on an encoding deficit, retrieval, and time in working memory: Evidence from once-presented words. Memory, 6, 37-65.

Challis, B. H. (1993). Spacing effects on cued-memory tests depend on level of processing. Journal of Experimental Psychology: Learning, Memory, \& Cognition, 19, 389-396.

Dempster, F. (1996). Distributing and managing the conditions of encoding and practice. In E. L. Bjork \& R. A. Bjork (Eds.), Memory (pp. 317-344). San Diego: Academic Press.

Elmes, D. G., Chapman, P. F., \& Selig, C. W. (1984). Role of mood and connotation in the spacing effect. Bulletin of the Psychonomic Society, 22, 186-188.

Elmes, D. G., Dye, C. J., \& Herdelin, N. J. (1983). What is the role of affect in the spacing effect? Memory \& Cognition, 11, 144-151.

GLENBERG, A. M. (1976). Monotonic and nonmonotonic lag effects in paired-associate and recognition memory paradigms. Journal of Verbal Learning \& Verbal Behavior, 15, 1-16.
GLENBERG, A. M. (1977). Influences of retrieval processes on the spacing effect of free recall. Journal of Experimental Psychology: Human Learning \& Memory, 3, 282-294.

Glenberg, A. M. (1979). Component-levels theory of the effects of spacing of repetitions on recall and recognition. Memory \& Cognition, 7, 95-112.

GreENE, R. L. (1989). Spacing effects in memory: Evidence for a twoprocess account. Journal of Experimental Psychology: Learning, Memory, \& Cognition, 15, 371-377.

JACOBY, L. L. (1978). On interpreting the effects of repetition: Solving a problem versus remembering a solution. Journal of Verbal Learning \& Verbal Behavior, 17, 649-667.

Kahana, M. J., \& Greene, R. L. (1993). Effects of spacing on memory for homogeneous lists. Journal of Experimental Psychology: Learning, Memory, \& Cognition, 19, 159-162.

MadigAn, S. A. (1969). Intraserial repetition and coding processes in free recall. Journal of Verbal Learning \& Verbal Behavior, 8, 828835.

Melton, A. W. (1970). The situation with respect to the spacing of repetitions and memory. Journal of Verbal Learning \& Verbal Behavior, 9, 596-606.

Shaughnessy, J. J., Zimmerman, J., \& Underwood, B. J. (1972). Further evidence on the MP-DP effect in free recall learning. Journal of Verbal Learning \& Verbal Behavior, 11, 1-12.

Thios, S., \& D' Agostino, P. R. (1976). Effects of repetition as a function of study-phase retrieval. Journal of Verbal Learning \& Verbal Behavior, 15, 529-537.

Toppino, T. C., \& Bloom, L. C. (2002). The spacing effect, free recall, and two-process theory: A closer look. Journal of Experimental Psychology: Learning, Memory, \& Cognition, 28, 437-444.

UNDERWOOD, B. J. (1969). Some correlates of item repetition in freerecall learning. Journal of Verbal Learning \& Verbal Behavior, 8, 8394.

UNDERWOOD, B. J. (1970). A breakdown of the total time law in freerecall learning. Journal of Verbal Learning \& Verbal Behavior, 9, 573 580

WAUGH, N. C. (1963). Immediate memory as a function of repetition. Journal of Verbal Learning \& Verbal Behavior, 2, 107-112.

WiCKENS, D. D. (1972). Characteristics of word encoding. In A. W. Melton \& E. Martin (Eds.), Coding processes in human memory (pp. 191-215). Washington, DC: Winston.

WiXted, J. T., \& Rohrer, D. (1993). Proactive interference and the dynamics of free recall. Journal of Experimental Psychology: Learning, Memory, \& Cognition, 19, 1024-1039.

\section{NOTES}

1. In multiprocess explanations of the spacing effect (e.g., Greene, 1989), two or more of these mechanisms may be included within a single theory.

2. It turned out that in one of the structures for the twice-presented/ long-lag condition, occurrences of one spaced-repetition item were separated by nine other presentations. And, in one of the structures for the twice-presented/short-lag condition, occurrences of one spaced-repetition item were separated by only two other presentations.

3. Component-levels theory, the most sophisticated form of encodingvariability theory (e.g., Glenberg, 1976, 1977, 1979), predicts that under certain circumstances, long lags separating repetitions can lead to poorer performance than shorter lags. This is predicted to occur when retrieval conditions favor access to one occurrence of a repeated item over the other. However, it is not clear how such an account can be applied to the present results.

(Manuscript received October 18, 2001; revision accepted for publication February 2, 2002.) 